\title{
Effects of 10 Weeks Swimming and Creatine Supplementation on Blood Lactate and Muscle Fatigue of Mice
}

\author{
By Ricardo Cesar A. Ferreira* \\ Wendel S. Fernandes ${ }^{t}$ \\ Fabiano Souza Barros ${ }^{t}$ \\ Wellington Ribeiro ${ }^{+}$
}

\begin{abstract}
Several ergogenic resources have been used by athletes to improve performance in their respective activities and succeed in their competitive events, or just with a esthetic purpose by increasing strength and rapid weight loss, a common practice in the clubs and fitness gyms.

Many of these supplements promise immediate solutions for those seeking hypertrophy, decreased body fat, increase strength, endurance, and decrease fatigue. Objectives: This research study the effect of creatine on physical activity in mice submitted to swimming. And Purpose to check the potential of Creatine in the possible reduction of fatigue using an electrical stimulator and getting data from tetanus contraction through eletrofisiográficos record, Also analyze the plasma level of animal lactate as physiological assessment parameter and enhance performance predictor tool and respective differences between groups. Methods: The animals were subjected to endurance exercise training for 10 weeks, achieved through swimming sessions (30 minutes / day for three days a week, Mondays, Wednesdays and Fridays, always held in the afternoon). Results: It was observed that the creatine groups shows significantly different values when compared to the others groups. Conclusion: Concluded that creatine associated with physical training promoted better muscle fatigue tolerance and just ten weeks physical activity can improve this physical condition.
\end{abstract}

Keywords: creatine, swimming, fatigue.

\section{Introduction}

Various tactics have been used by athletes in an attempt to boost performance and sports performance, through the physical training or using ergogenic drugs. A growing number of athletes have been using supplementation crediting at it their improvement in their respective sports ${ }^{1-2}$. Most supplements contain many ingredients in its formula to produce ergogenic effects. When ingested, these ingredients may work synergistically

\footnotetext{
${ }^{*}$ Universidade Paulista, Brazil.

${ }^{\dagger}$ Universidade Paulista, Brazil.

* Universidade do Vale do Paraíba, Brazil.

+ Universidade do Vale do Paraíba, Brazil.
} 
increasing various aspects of exercise performance ${ }^{3,14}$. The ergogenics are substances used to enhance physical performance and since the dietary supplement was legalized the best-selling as an ergogenic aid is creatine. After the 1996 Olympics, creatine supplementation has become very popular among athletes ${ }^{4}$.

Exercise promotes an instant boost of energy demand, thus causing imbalance of body homeostasis ${ }^{5-13}$, and the possibility to increase the performance, be it physical or mental. To Oliveira et al. (2014) skeletal muscle has the ability to modify their structural and functional properties, such as the phenotype profile and / or size of muscle fiber area in accordance with the stimuli it receives from the external medium, this is called adaptive capacity muscle plasticity. This fact has aroused interest, according to the importance they may have on athletic performance. Elucidate the mechanisms of fatigue and stress and ways to avoid them if necessary ${ }^{1-12}$.

The measurement of isometric force of tetanic muscle contraction can be considered an important tool for the assessment of muscle strength and fatigue ${ }^{6}$. With the tibialis anterior muscle preparation technique and electrical stimulation directly to the sciatic nerve, you can keep the innervation and vascularization of the muscle during the experiment maintaining its physiological properties.

\section{Materials and Methods}

This study was conducted by research project approval sent to the Ethics Committee on Animal Research / IPD-UNIVAP under the A02 protocol / CEUA / 2013.

\section{Animals}

In the present study, male mice were used (Swiss) with 06 weeks of age with different weighing $20 \pm 3 \mathrm{~g}$. The animals were kept in plastic boxes (five animals per cage) with temperature $\left(22-25^{\circ} \mathrm{C}\right)$, relative humidity $(40-60 \%)$ and controlled photoperiod (cycle of 12 hours light / dark) being fed with water and food ad libitum.

The animals were divided into six groups, with five animals per group as listed below:

Group 1 (SED): sedentary animals that receive restricted diet water and commercial balanced feed.

Group 2 (SED + CR): sedentary animals that besides water and food will receive creatine supplementation.

Group 3 (SWI): Animals exercised in the pool who will receive restricted diet water and balanced diet.

Group 4 (SWI + CR): Animals exercised in the pool which will receive creatine supplementation in addition to water and balanced diet. 
The animals received creatine monohydrate dissolved in water orally with needle aid gavage technique to administer liquid food directly into the stomach daily for 10 weeks. In the 1 st week of supplementation (phase load or overload), the dose of creatine was $0.3 \mathrm{~g}$ body weight of each animal. In the following weeks (maintenance phase), the dose was $0.075 \mathrm{~g}$, equivalent to a dosage of $20 \mathrm{~g} /$ day for a person weighing $70 \mathrm{~kg}$, which shows maximum effect within five days.

Figure 1. Technique used to Deposit Liquid Directly into the Esophagus known as Gavage

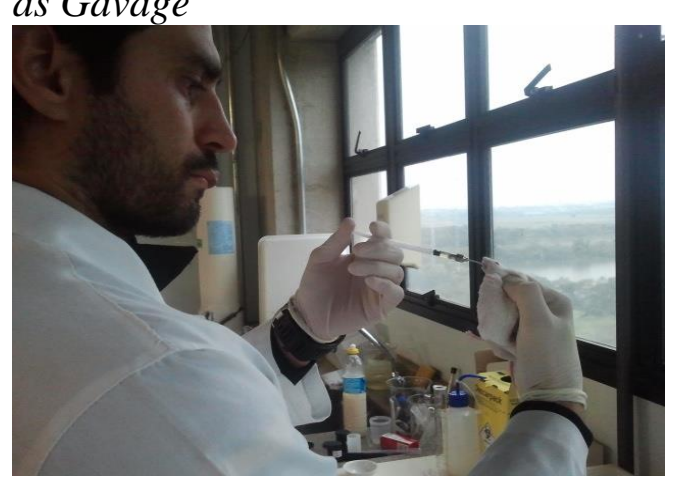

Experimental Protocol

The animals were subjected to endurance exercise training for 10 weeks, achieved through swimming sessions (30 minutes / day for three days a week, Mondays, Wednesdays and Fridays, always held in the afternoon).

The training was conducted overloaded $80 \%$ of the maximum load for each animal. The maximum load was determined by the Maximum Load Test ${ }^{7}$ (Osorio et al., 2003). The material used for making the loads were heavy lead ball on a precision scale (Belß) and attached to the animal body through individual elastic vests. To perform the physical activity protocol was used a fiber tank with dimensions of $100 \mathrm{~cm}$ (length) x $50 \mathrm{~cm}$ (width) x $60 \mathrm{~cm}$ (height). Drinking water was used, which was replaced after each training session. The water temperature was set at $33^{\circ} \mathrm{C}$ by thermostat installed along the side of the tank and monitored by a manual mercury thermometer throughout the training session. The water depth was set at $40 \mathrm{~cm}$, enough so that the animals did not support the distal end of the tail at the bottom in the tank. Swim sessions were conducted collectively, so that the five animals of the same group swam at the same time. After the end of each session the animals were removed from the water, wiped dry manually and placed under bathroom light.

\section{Maximum Load Testing for Swimming}

The first Maximum Load Test (MCT) was performed 24 hours after the adjustment period, in all animals swimming groups, individually. The animals were weighed and the test started with an overload of $1 \%$ of body weight ${ }^{7}$. For each phase three minutes with a 1 minute recovery was added over $1 \%$ filler 
and so on until the animal can not stay on the water surface. From the immersion were timed $8 / 2$. When the animal can not return to the surface after this period will be characterized that this charge is your limit, this is removed from the water and the previous charge was considered the maximum load of the respective animal.

\section{Maximum Contraction Tests}

In animal models, the anterior tibial muscle is significant research interest due to their relatively large size, the sciatic nerve innervation and its mixed composition type of muscle fibers by the features have been widely used as a research tool ${ }^{6-11}$.

The animals were submitted to a muscle contraction test in isometric force transducer in the week following the end of the swimming and supplemental training, not to characterize the detraining or mitigate the effects this could provide. All animals were anesthetized and sedated, after checking the reflexes and confirmed that the animal no longer respond to stimuli started the surgical procedure. We dissected the right tibialis muscle and an incision was made to locate the sciatic nerve, so located, this was connected to an electrode to receive electrical impulses ${ }^{10-11}$. The tendon was also dissected and connected to electrophysiograph to record the contractions performed by the muscle. Importantly, the muscle and tendons and the nerve remained moistened by saline.

Figure 2. Electrode Connected to the Animal's Sciatic Nerve

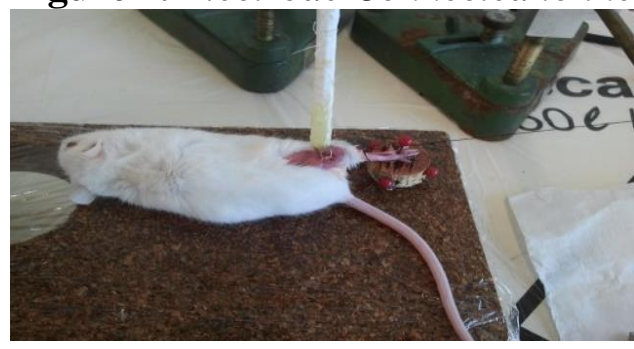

Figure above allows us to observe the time of the procedure where the electrode is connected to the sciatic nerve and the tendon tied to the transducer.

Stimulation was performed by pulses of $1 \mathrm{~Hz}$, after establishing the minimum voltage at which the muscle showed response, the voltage is gradually increased until we find the maximal voltage with stimulus of 2 minutes. And tetanic contractions of the muscle were stimulated using an isometric transducer, to induce tetanic contraction. The frequency was increased to $50 \mathrm{~Hz}$ for a period of 10 seconds. Muscle fatigue was characterized by inability to maintain muscle contraction.

The responses to stimuli were recorded in a 7070 GEMINI electrophysiograph of UGO BASILE, the speed of the paper used for the records was $005 \mathrm{~mm} / \mathrm{s}$ and $300 \mathrm{~mm} / \mathrm{s}$ respectively. 


\section{Analysis of Lactate}

Blood samples $(\sim 25 \mu \mathrm{l})$ were obtained by the caudal end section of each animal and placed in test strips for the quantification of lactate (BMLactate $\AA$ ). Then these test strips containing the samples were immediately introduced in Accutrend ${ }^{\circledR}$ Lactate portable analyzer for determination of lactate concentrations, respectively. Samples were collected before the muscle contractions and the following three minutes after the third and final tetanic contraction to obtain the maximum peak lactate concentration.

\section{Anesthesia Protocol and Sacrifice of Animals}

The animals were sedated and anesthetized by intraperitoneal (ip) injection of ketamine and xylazine $10 \% 2 \%$ at a dosage of 50 microliters each.

Later the animal was positioned on the operating table in the supine position, then an incision was made in the anterior tibial height right animal for the identification and isolation of the sciatic nerve, where the electrodes for electrical stimulation were connected after this procedure has been identified and isolated the tendon of the right paw of the animal, which was connected to a surgical line and is tied to electrophysiograph, As the electrical stimuli were given muscle contraction and consequently tendon and collected by the device $^{11}$. Upon completion of the blood collection for lactate analysis was used syringes $1 \mathrm{ml} / \mathrm{cc}$ and needles $0.45 \times 13 \mathrm{~mm}$ for the sacrifice of animals, still under anesthesia through intracardiac injection of potassium chloride (KCL) at $20 \%$.

\section{Statistical Analysis}

Statistical analysis was performed using the statistical software Graphpad Instat (Graphpad Software Inc. Version 6.0). The results were demonstrated through tests and ANOVA multiple comparison Tukey-Kramer, were applied to assess the mean values of different groups, differences were considered significant if $\mathrm{p}<0.05$. For comparative weight values $(\mathrm{g})$ we used the $\mathrm{T}$ test were considered significant differences at $\mathrm{p}<0.05$.

\section{Results and Discussion}

Figure 1 shows the graph corresponding to the average area under the curve tetanus $(\mathrm{cm} 2)$ for 10 seconds of each corresponding induced contraction and the muscle's ability to resist fatigue and to keep at maximum shrinkage tension or next higher during this time possible ${ }^{9-14}$. It was observed that the SED + CR group had significantly different values when compared to the SED Group and the Group SWI + CR showed significant differences for the SED groups 
Figure 1. Area Under the Curve for 10 Seconds Tetanic Contraction in Swiss Mice for the Different Experimental Groups. The Data Represent the Mean \pm $S D ; n=5 ; * P<0.05$ and Compared with the Response Obtained in the Group: Control (SED); \# P <0.05 Compared with the Response Obtained in the Group that underwent Swimming (NAT). (ANOVA, followed by TukeyKramer multiple comparison test)

\section{A rea}

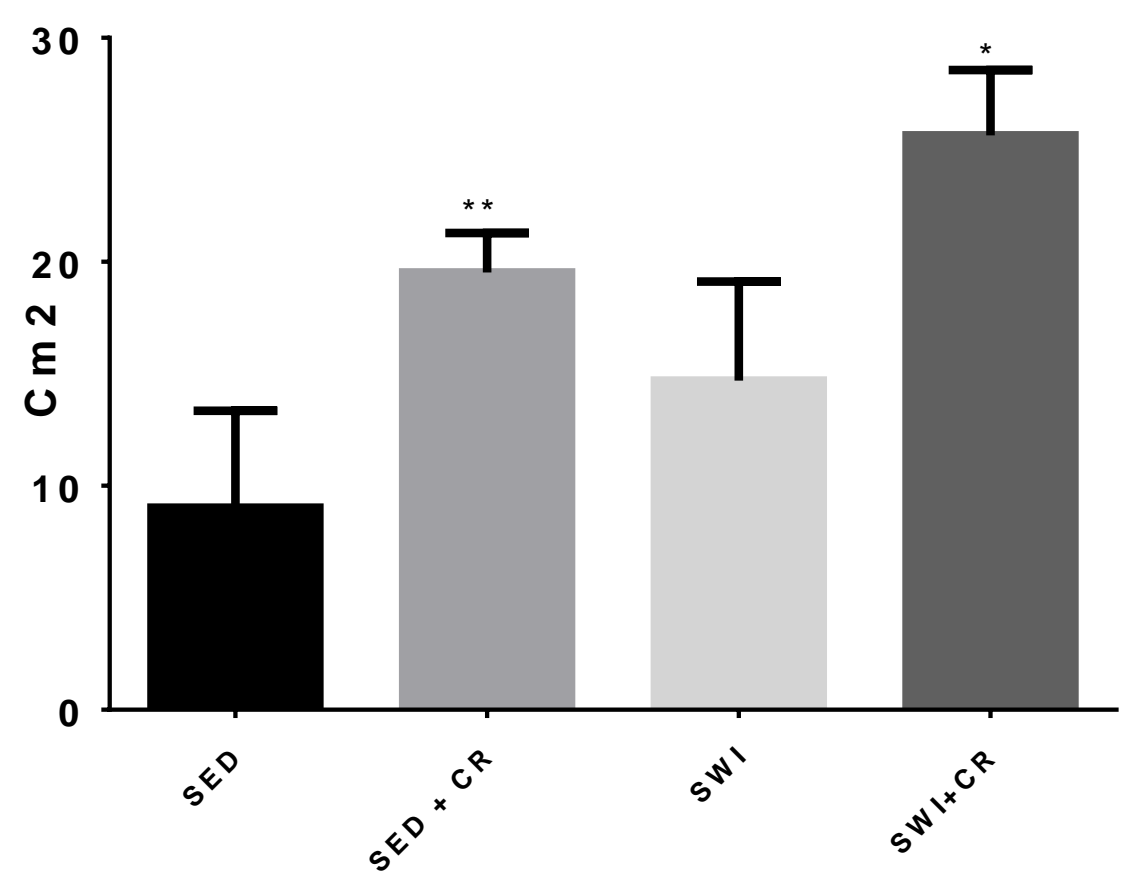

Figure 2, refers to analyze of lactate, where the sedentary groups had higher lactate levels than the other groups. Overall the two groups of sedentary, having ingested or not supplements, had higher mean values than the groups who exercised, and the swimming group + creatine showed lower acidosis than all the others put these values was not statistically significant. 
Figure 2. Graph of the Blood Lactate Concentration in Swiss Mice after Muscle Contraction Test the Different Experimental Groups. The Data Represent the Mean $\pm S D ; n=5 ; * P<0.05$ Compared with the Response Obtained in Group Control (DES); (ANOVA followed by Tukey-Kramer multiple comparison test)

\section{Lactato}

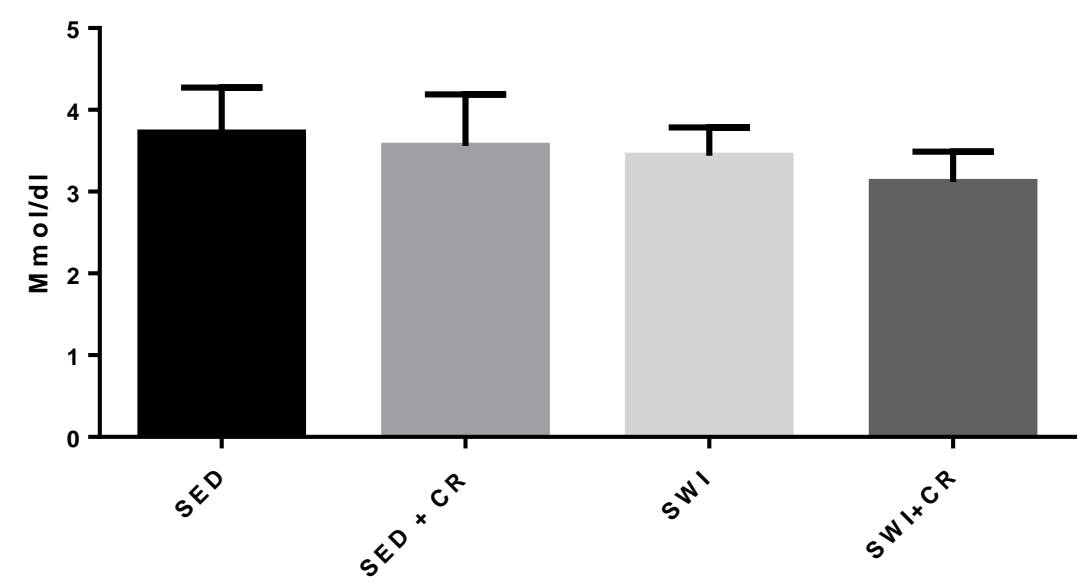

The study investigated by tetanic muscle contraction by electrical stimulation, muscle fatigue of animals submitted to physical activity and supplementation. The modality was proposed by this feature a swimming endurance exercise and try to frequent and regular use of leg muscles, since the animals have to stay on the water surface.

These data were obtained from three electrically induced tetanic contractions of the tibialis anterior muscle.

Regarding the mean area under the curve tetanus. The results show that the SED group, representing the control group had significantly lower average to Sedentary who used creatine as an ergogenic aid, SED + CR.

For the same variable SWI + CR group, group that performed swimming as physical activity associated with the use of Creatine, averaged higher and significant differences for SED and SWI, with no difference between the two groups that ingested creatine. These results seem to demonstrate a possible contribution of supplementation with creatine in delaying the process of muscle fatigue.

Finally about to the graph corresponding to the sample of blood lactate, trained groups showed lower concentration values than their respective controls. What could highlight the importance of physical activity for increasing aerobic capacity, slowing acidosis accumulation, thus providing greater contractile capacity of muscle in a period of time also higher. However, all of these lactate values were not statistically significant. 


\section{Conclusion}

Concluded that, at least under our experimental conditions, supplementation with creatine associated with physical training promoted better muscle fatigue tolerance and just ten weeks physical ativity can improve this physical condition. The indiscriminate use of supplements can in addition, to cause side effects (addiction, risk of acute myocardial infarction, behavioral changes), not achieve the results promised in their advertising. Further studies are needed to disseminate that knowledge and awareness of health professionals in this area on risks of these products.

\section{References}

Oliveira, D. R. et al. Efeito da natação associada a diferentes tratamentos sobre o músculo sóleo de ratos: estudo histológico e morfométrico. Rev. Bras. Med. Esporte., v.20, n.1, p.74-77, 2014.

Hoffman, J. R. et al. Examination of a pre-exercise, high energy supplement on exercise performance. Journal of the International Society of Sports, V 6, p 2, Jan 2009.

Spradley, B. D. et al. Ingesting a pre-workout supplement containing caffeine, Bvitamins, amino acids, creatine, and beta-alanine before exercise delays fatigue while improving reaction time and muscular endurance. Nutrition \& Metabolism, v.9, n.28, 2012.

Tokish, J. M. Kocher, M. S. Hawkins, R. J. Ergogenic aids: a review of basic science, performance, side effects, and status in sports. Am. J. Sports Med., v.32, n. 6, p.1543-1553, 2004.

Caruso, F. C. R. et al. Efeitos do treinamento de força sobre a função pancreática e a sensibilidade tecidual à insulina em ratos. Arq. Ciênc. Saúde UNIPAR, vol 14, n 3, 2010.

Shin, R. H. et al. Isometric tetanic force measurement method of the tibialis anterior in the rat. Microsurgery. v.28, n.6, p.452-7, 2008.

Osório, R. A. L. et al. Swimming of pregnant rats at different water temperatures. Comp. Biochem. Phisiol. A. Mol. Integr. Phisiol., v.135, n.4, p.605-611, 2003.

Rohlfs, I. C. P. M. et al. Relação da síndrome do excesso de treinamento com estresse, fadiga e serotonina. Rev. Bras. Med. Esporte .V11, n.6, p.367-372, 2005.

Terjung, R. L. et al. The physiological and health effects of oral creatine supplementation. Med. Sci. Sports Exerc., v.32, p.706-717, 2000.

Antonio, J.; Ciccone, V.; The effects of pre versus post workout supplementation of creatine monohydrate on body composition and strength. Journal on the International Society of Sports Nutrition. v.36. n. 10. p. 1-8. 2013.

Gouvea H.A. et al. Analise eletrofisiografica e histomorfologica do músculo tibial anterior de ratos submetidos a treinamento aeróbio com natação. Fit Perf J. 2009 Jan-fev 8 (1) p 49-55.

Volek, J. S.; Rawson, E. S. Scientific basis and practical aspects of creatine supplementation for athletes. Nutrition, v20, p.609-614, 2004. 
Syrotuik, D.G.; et al. Absolute and relative strength performance following creatine monohydrate supplementation combined with periodized resistance training. $J$ Strength Cond Res 2000; v14, 182.

Terjung, R.L. et al. The physiological and health effects of oral creatine supplementation. Med Sci Sports Exerc 2000; v32, p.706-717.

Theodorou, A. S. et al. Effects of acute creatine loading with or without carbohydrate on repeated bouts of maximal swimming in high-performance swimmers. $J$. Strength Cond. Res. v. 19, n. 2, p. 265-269, 2005. 
